\title{
Solitary cerebral metastasis from a papillary carcinoma of the thyroid
}

\author{
A.W.G. Goolden, J.E. Mclaughlin, A.R. Valentine and C. Pease' \\ The Endocrine Unit, Department of Histopathology and Department of Radiology, The Royal Free \\ Hospital, London NW3 3QG and ${ }^{1}$ St. Andrew's Hospital, Billericay, Essex CM12 OBH, UK.
}

\begin{abstract}
Summary: A woman aged 52 was treated with radioactive iodine for a papillary carcinoma of the thyroid. Four years later she developed signs and symptoms of an intracranial space occupying lesion. A computed tomographic scan showed a mass in the right posterior frontal region. Although she was suspected of having metastatic disease a definite diagnosis was not established until she died 6 months later when post-mortem examination confirmed that she had a cerebral metastasis from a papillary carcinoma of the thyroid. There was no evidence of metastatic disease elsewhere in the body.

Cerebral metastases from papillary carcinoma of the thyroid are uncommon but may occur in patients who have metastases in bones or lungs. A search of the literature has revealed only two patients with solitary cerebral metastases.
\end{abstract}

\section{Introduction}

Papillary carcinoma of the thyroid is the commonest type of thyroid tumour constituting between 30 and $70 \%$ of all thyroid carcinomas. There is a wide age range. In younger patients the tumour is nearly always confined to the neck with or without spread to the cervical lymph nodes. The prognosis in general is good - better than for any of the other types of thyroid tumour - but in older patients papillary carcinoma behaves in a more aggressive manner and may give rise to distant metastases. Such metastases are commonly sited in the lungs or bones. We report here a patient with papillary carcinoma of the thyroid who had a solitary metastasis in the brain.

\section{Case report}

At the beginning of 1984 a woman aged 52 noticed increasing hoarseness of her voice. On examination she was found to have a hard mass on the left side of her neck and a paralysed left vocal cord. Surgical exploration in February 1984 revealed a tumour replacing the left lobe and isthmus of the thyroid gland. It was infiltrating the strap muscles and was firmly fixed to the trachea. Biopsy was performed but no attempt was made to remove the tumour. Histological examination showed a locally invasive papillary carcinoma of the thyroid which, as is frequently the case with papillary carcinoma, was predominantly of follicular pattern.

Correspondence: A.W.G. Goolden, F.R.C.P., F.R.C.R. Orchard House, Summerhouse Lane, Aldenham, Herts WD2 8DL, UK.

Accepted: 26 July 1989
She was referred to the Royal Free Hospital for further management. A thyroid scan showed a normal right lobe but diminished activity in the region of the left lobe. Thyroid uptake at 24 hours was $15 \%$. It was decided to treat her with radioactive iodine $\left({ }^{131} \mathrm{I}\right)$. She was accordingly given $120 \mathrm{mCi}(4.3 \mathrm{GBq})$ of ${ }^{131} \mathrm{I}$ in March 1984 with the intent of first ablating normal thyroid tissue. She developed radiation thyroiditis 3 days after this treatment. Three months later a scan showed activity just above and to the left of the suprasternal notch with a 24 -hour uptake of $0.3 \%$. She was given a second therapeutic dose of $150 \mathrm{mCi}$ (4.5 GBq) of ${ }^{131} \mathrm{I}$ in June 1984 . She was subsequently maintained on $200 \mu \mathrm{g}$ of thyroxine daily.

She remained well for the next 4 years. Throughout this time there was some induration on the left side of her neck but no tumour could be identified. Her progress was monitored with serial measurements of serum thyroglobulin $(\mathrm{Tg})$. This never became normal $(<0.1 \mu \mathrm{g} / \mathrm{l})$, varying between 11 and $27 \mu \mathrm{g} / \mathrm{l}$. The source of the $\mathrm{Tg}$ was assumed to be residual primary tumour, but in retrospect could have been the metastasis.

When seen in March 1988 she complained of drowsiness, confusion and weakness of her left hand. On examination she had dysdiadokokinesis of the left hand and loss of power in the left hand and arm. The signs and symptoms suggested an intracranial space occupying lesion. A computed tomographic (CT) scan (Figure 1) showed a lobulated mass in the right posterior frontal region immediately above the anterior part of the lateral ventricle. The findings were thought to be compatible with metastatic disease. A chest X-ray was 


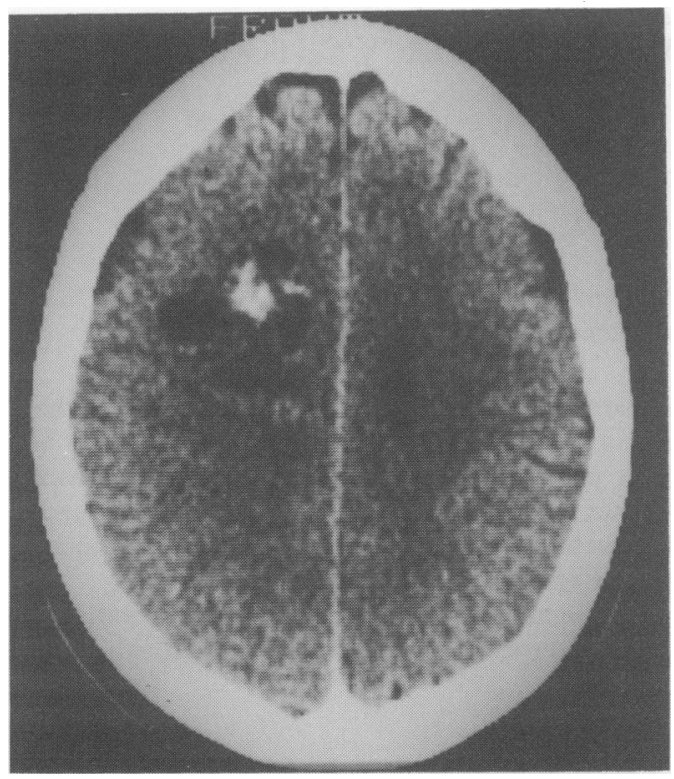

Figure 1 Computer tomogram of brain showing a lobulated mass impressing the ventricular roof in the deep right fronto-parietal region. This enhanced image suggests that the mass has cystic (non-enhancing) and solid (enhancing) components.

normal. Liver function tests were normal. A mammogram showed no evidence of breast carcinoma. A test dose of $5.0 \mathrm{mCi}(185 \mathrm{MBq})$ of ${ }^{131} \mathrm{I}$ was given 24 hours after an intramuscular injection of 5 units of bovine thyroid stimulating hormone (TSH) and a whole body scan was performed 72 hours later. The patient was given exogenous TSH in preference to allowing her to become hypothyroid because the retention of fluid which occurs in hypothyroidism would undoubtedly have resulted in a deterioration of her neurological condition. Scanning (performed under optimal conditions of timing and dose) showed no activity in abnormal sites and in particular no activity in the neck or brain. It was concluded that the patient had metastatic disease but there was some doubt as to whether this was secondary to the thyroid tumour. It was clearly unsatisfactory not having a tissue diagnosis but the neurosurgical opinion was that biopsy carried some risk and that it was not justified unless it was going to alter her treatment. She was given a course of external irradiation in the hope that this would retard the progress of her disease. She received a dose of $3600 \mathrm{cGy}$ in 12 fractions.

By July her condition had deteriorated. She could do very little with her left arm and had largely lost the use of her left leg. A CT scan showed the ventricular system to be slightly larger than on the previous examination but the metastic lesion had not changed. Her condition continued to deteriorate. She died 6 months after presentation.

Post-mortem examination showed the cause of death to be a massive embolus occluding the right main pulmonary artery. The thyroid gland was represented as a thin fibrous strip. There was no evidence of regional lymph node involvement. Sectioning of the brain showed a well circumscribed solid and cystic tumour mass within the right fronto-parietal white matter. The mass contained small areas of apparent necrosis. It measured $3 \mathrm{~cm}$ in its maximum dimension and extended into the outer margin of the right anterior horn of the lateral ventricle. There was no evidence of metastatic disease elsewhere in the body.

Histological examination showed the tumour to be composed of multiple papillary processes showing great variation in size. Small foci of calcification (psammoma bodies) were noted. In some areas there were small follicular structures containing colloidal substance (Figure 2). Immunohistochemical staining showed intracellular thyroglobulin in some cells. The appearances were those of metastatic papillary carcinoma of the thyroid.

\section{Discussion}

McConahy et al. ${ }^{1}$ reviewed all patients with papillary carcinoma of the thyroid treated at the Mayo Clinic between 1946 and 1970. In a series of 859 patients $11(1.3 \%)$ were identified as having cerebral metastases. Høie et al. ${ }^{2}$ used the Cancer Registry in Norway to find out the incidence of distant metastases in patients with papillary carcinoma of the thyroid. In a series of 731 patients, registered between 1956 and 1978, 91 developed distant metastases. Seventy three of these patients had intrathoracic metastases, 17 skeletal metastases and $9(1.2 \%)$ cerebral metastases. In both the above series the patients who developed cerebral metastases also had metastases in lungs or bones or in both sites.

As Parker et al. ${ }^{3}$ have pointed out, cerebral metastases from papillary carcinoma of the thyroid are uncommon. These authors reported two patients where the tumour had metastasized to the brain. One had metastases elsewhere. The second one did not, and this would appear to be the first recorded instance of a solitary cerebral metastasis secondary to a primary papillary carcinoma of the thyroid. A search of the literature has revealed only one other case, a patient with an occult primary carcinoma of the thyroid. ${ }^{4}$

The patient whose case history is described here is another example of a solitary cerebral metastasis from a papillary carcinoma of the thyroid. The rarity of this condition made us reluctant to make what turned out to be the correct diagnosis which 


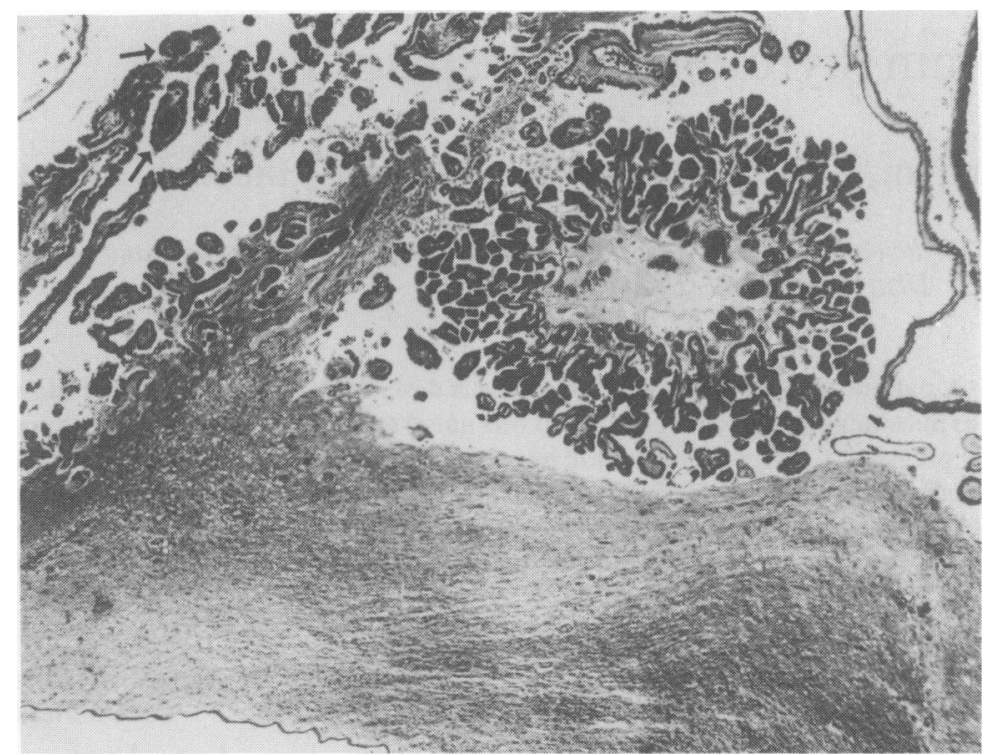

Figure 2 Cerebral metastasis showing papillary thyroid carcinoma. An occasional psammoma body is present (arrow). $\mathrm{H}$ and $\mathrm{E}(\times 10)$.

was only confirmed after a post-mortem examination had been carried out. Non-iodine-concentrating metastases have been identified by giving the patient labelled anti-thyroglobulin antibodies and subsequently doing a scan ${ }^{5}$ but this technique is not currently available.

Histological examination of the metastasis showed some areas where there were follicular structures containing colloidal substance and some cells were seen to be producing thyrogobulin, the latter probably accounting for the elevated levels of thyroglobulin in the serum. It might seem surprising that the metastasis did not concentrate iodine. However, although histological appearances provide some indication of whether or not a tumour is likely to take up iodine there is not always a good correlation between structure and function.

The primary tumour did presumably contain functioning thyroid tissue because it resolved with no treatment other than ${ }^{131}$ I. It might be expected that metastases from a functioning primary tumour would behave in the same way as the parent tissue with respect to iodine concentration but this is not always the case. Metastases are often less well differentiated than the primary. Comparison of sections of the primary tumour and the metastasis in this patient suggest that the follicular foci in the metastasis were much less prominent.

Treatment of a cerebral metastasis from a differentiated carcinoma of the thyroid is a problem if the metastasis does not concentrate ${ }^{131} I$. This patient's tumour clearly did not respond to external irradiation. One of the patients described by Parker et al. ${ }^{3}$ was treated by surgical excision, an implant using ${ }^{125}$ I seeds and external irradiation but this method of treatment would be of questionable value in a patient who already had quite severe brain damage.

\section{Acknowledgement}

We wish to thank Miss Elizabeth Black for performing the thyroglobulin assays.

\section{References}

1. McConahey, W.M., Hay, I.D., Woolner, L.B. \& Taylor, W.F. Papillary thyroid cancer treated at the Mayo Clinic, 1946 through 1970: initial manifestations, pathologic findings, therapy and outcome. Mayo Clin Proc 1986, 61: 978-996.

2. Høie, J., Stenwig, A.E., Kullman, G. \& Lindegaard, M. Distant metastases in papillary thyroid cancer. Cancer 1988 , 61: $1-6$.

3. Parker, L.N., Wu, S.Y. \& Kim, D. Recurrence of a papillary carcinoma presenting as a focal neurological deficit. Arch Intern Med 1986, 146: 1985-1987.
4. Michie, H.R., O'Bryan-Tear, C.G., Marsh, H. \& Glazer, G. Cerebral metastasis from occult papillary carcinoma of the thyroid. Br J Surg 1987, 74: 647.

5. Fairweather, D.S., Bradwell, A.R., Watson-James, S.F., Dykes, P.W., Chandler, S. \& Hoffenberg, R. Detection of thyroid tumours using radio-labelled antithyroglobulin. Clin Endocrinol 1983, 18: 563-570. 